\title{
Patient Radiation Dose Assessment during Fluoroscopic Procedures: A Survey to Propose Local Diagnostic Reference Levels for Selected Facilities
}

\author{
Otoo. J J $^{*_{1}}$, Boadu. $\mathrm{M}^{2}$, Sosu. E ${ }^{3}$, Inkoom. $\mathrm{S}^{4}$ \\ ${ }^{*}$ Radiation Protection Institute, Ghana Atomic Energy commission, Accra, Ghana \\ ${ }^{2}$ School of Nuclear and Allied Sciences, University of Ghana, Accra, Ghana \\ 3.4Radiological and Medical Sciences Research Sciences Institutes, Accra, Ghana
}

\begin{abstract}
Article Info

Volume 7, Issue 4

Page Number: 05-11

Publication Issue :

July-August-2020

\section{Article History}

The primary aim of this work was to propose local diagnostic reference levels (LDRLs) for fluoroscopy examinations in two selected radiological imaging facilities in Ghana for subsequent improvement of optimization. DRLs was established for the frequently performed procedures, which includes, hysterosalpingogram (HSG), urethrogram (Utg) and barium swallow (BaS) examinations. The DRLs for KAP and screening time values estimated for hysterosalpingography was $6.0 \mathrm{~Gy} . \mathrm{cm}^{2}$ and 0.60 minutes, ; Barium swallow was $12.1 \mathrm{~Gy} . \mathrm{cm}^{2}$ and 1.4 minutes ; and urethrogram studies was $7.0 \mathrm{~Gy} . \mathrm{cm}^{2}$ and 0.7 minutes for Facility A . Whiles for Facility B, the DRLs for KAP and screening time values estimated for hysterosalpingography was $4.1 \mathrm{~Gy} . \mathrm{cm}^{2}$ and 0.50 minutes; ; Barium swallow was 11.2 Gy.cm² and 1.2 minutes ; and urethrogram studies was $6.5 \mathrm{~Gy} . \mathrm{cm}^{2}$ and 0.7 minutes.
\end{abstract}

Accepted : 01 July 2020

Published : 07 July 2020
Keywords: Diagnostic Reference Levels (DRLs); Fluoroscopic examinations; Kerma- Area Product (KAP)

\section{INTRODUCTION}

Fluoroscopy is a real-time X-ray imaging technique that is used in the clinical settings for diagnosis, allowing the physician to assess dynamic functions of the human anatomy [1]. It makes use of $\mathrm{X}$-rays to achieve real -time moving images of an object (body), since $\mathrm{X}$-rays, are a form of ionizing radiation, there are risks accompanying their use in the practice of fluoroscopy [1]. X-ray examinations involving fluoroscopy, mostly those done in cardiology and gastroenterology in addition to angiography and interventional procedures, contribute significantly to the overall collective dose due to medical exposure even if their rate of recurrence is moderately low [2]. Diagnostic Reference Levels (DRLs) are not proposed as absolute dose limits, but used relatively for the optimization of radiation doses and a means for quality assurance in diagnostic and/or therapeutic (interventional) procedures. Where procedures regularly exceed the DRL, investigation is necessary.For fluoroscopically guided examinations, 
DRLs in principle can be employed in the management of patient doses to avoid unnecessary stochastic effects.

Establishing of Investigative Reference Levels (IRL) is to facilitate the optimization of the image quality and dose in radiology, according to the (ALARA) principle, which states, doses must be kept 'As Low As Reasonably Achievable' [3]. Several national and international bodies promote this approach/philosophy for example the International Commission on Radiological Protection (ICRP). The theory of DRLs is of central significance in the management of radiation doses delivered to the patient in both interventional and diagnostic radiology [4].

Currently there are no nationally or regionally recommended DRLs for fluoroscopy procedures for use in Ghana. In this research, local diagnostic reference levels (LDRLs) at major public hospitals were set for a wide range of detailed diagnostic fluoroscopic procedures. DRLs are set in terms of the practical dose qualities used to monitor practice. For fluoroscopy procedures kerma-area product (KAP) or dose area product (DAP) is the recommended primary DRL quantity. Number of images and fluoroscopy time which are provided on the DAP console are recommended as valuable additional DRL quantities [5]. DAP has been shown to correlate well using the total energy given to a patient, which is associated to the effective dose and therefore to overall cancer risk. The principal objective of this research was to separately propose Local Diagnostic Reference Levels for two major referral hospitals in Accra - Ridge Hospital and Korle- $\mathrm{Bu}$ Teaching Hospital. The specific objectives include investigating the dose distribution levels of patient radiation dose received during fluoroscopy examinations and identifying if there is the need for optimization. Finally, to perform inter-comparison studies of the data collected with international DRLs.

\section{METHODS AND MATERIAL}

Prior to the commencement of data capture in this research, a series of Quality Control Tests were performed on two machines using a calibrated dose quantity kit manufactured by Piranha as shown in figure 1,(with specifications Type: Piranha 657 with serial number: CB2-15020088), calibrated in March 11, 2015 to assess whether the machine output is exactly what is expected. The tests performed were; Timer Accuracy, kVp Accuracy, mAs Linearity, Collimation Accuracy, Half value layer measurements, Tube Voltage, Exposure and Exposure Time Reproducibility. Quality control tests performed on the two-fluoroscopy machines were found to be the acceptable criteria according to well-established international protocols such as the AAPM. The research was done at two public hospitals, located in Accra - the capital in the Greater Accra Region of Ghana, between December 2017 and May 2018. To ensure confidentiality and anonymity of patient information, and patient identity, facilities were coded with alphabets. A prospective quantitative research method was adopted to obtain frequency of fluoroscopy examinations in the Radiology Department [6]. A calibrated KAP meter, Kerma XPlus iba dosimetry model 120-131 HS with serial number: 01A04042 was used to measure the KAP. In use, the KAP meter with all associated electronics was placed perpendicular to the central beam axis and in position to completely intercept the entire area of the $\mathrm{X}$-ray beam as shown in figure 2 [7]. KAP data values and screening time of the fluoroscopy procedure performed were obtained from the machine's console after each patient's examination. However, no additional adjustments or scan protocols were used for this research, to ensure the study reflected the actual normal practices in all the centres. The data obtained 
was statistically analysed using Microsoft Excel and results presented in descriptive statistics of tables, charts and graphs. In other to calculate the $75^{\text {th }}$ percentile, which is the value for the DRLs, the weighted mean equation was used;

$$
\text { Rank }=\frac{\text { Percentile }}{100} \times(\text { Number of patients }+
$$

1)

.......Eqn 1

$75^{\text {th }} \mathrm{P}=\min$ Rank $+[(\max$ Rank $-\min$ Rank $) \times$ Decimal value] Eqn 2



Figure 1: Piranha Kit

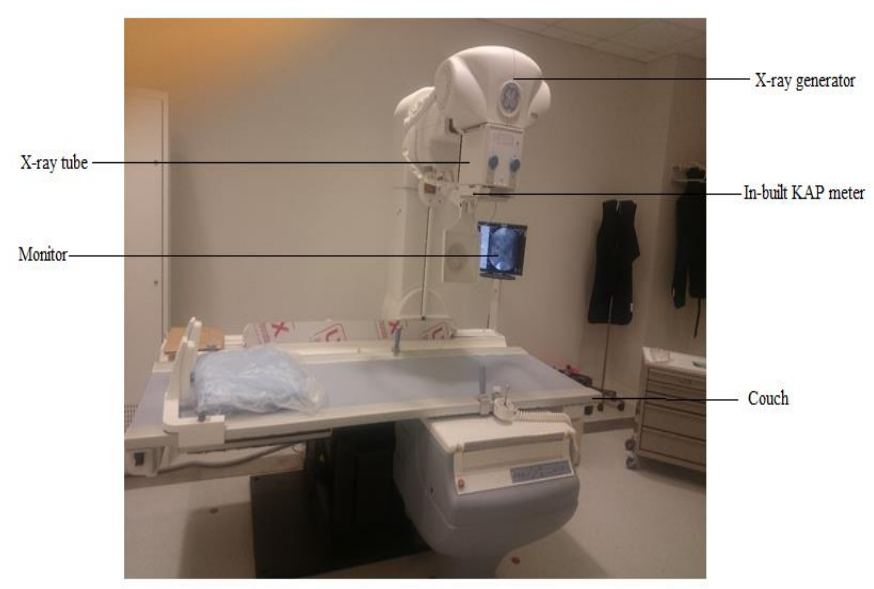

Figure 2 : Fluoroscopy Machine with in-built KAP meter

\section{III.RESULTS AND DISCUSSION}

At least 10 patients per examination were required for this research work. According to Vañó, et al, surveys to establish diagnostic reference levels should be based on measurable patient data, which should represent at least 10 patients per examination for each procedure [8]. A total sample size of 249 patients was collected. The distribution of patient sample for the study sites is described in table 1 .

Table 1: Distribution of Patient Sample

\begin{tabular}{cc}
\hline FACILITIES & $\begin{array}{c}\text { NUMBER OF } \\
\text { PATIENTS }\end{array}$ \\
\hline FACILITY A & 111 \\
FACILITY B & 138 \\
& \\
\hline
\end{tabular}

During the study, some of the fluoroscopy examination did not meet the requirement to estimate DRLs. At facility "A", 111 patient data was taken, out of which 98 patient data passed the acceptance criteria which comprised $\mathrm{HSG}=63.27 \%, \mathrm{BaS}=12.24 \%$ and $U \operatorname{tg}=24.49 \%$ of the accepted data.

However, for Facility "B", 82 patient data passed the acceptance criteria out of the 138 patient data collected. The accepted data was distributed as, 65.85\%, $19.51 \%$ and $14.63 \%$ for HSG, BaS and Utg examinations respectively.

\section{Proposing the Diagnostic Reference Levels (DRLS)}

Details of the descriptive statistics from the surveyed examinations are presented in Figures 3 and 4. The DRLs for the KAP and screening time for each fluoroscopy examination were calculated for both facilities. A comparative study was also done to compare KAP values and fluoroscopy screening time across the fluoroscopy facilities and other studies done in other countries. The individual facilities are denoted by the alphabets A and B in order to avoid mentioning facility names for confidentiality and anonymity purposes. 
Tables 2 and 3 represents 75th percentiles of the KAP values for a particular examination and the fluoroscopy screening time from the facilities surveyed. The 75 th percentiles imply that 75 percent of the examinations surveyed operate at or below the dose, values presented for all the categories of examination surveyed. These values represent values with which fluoroscopy practices in these facilities can be compared to and with recommended standards survey from other countries

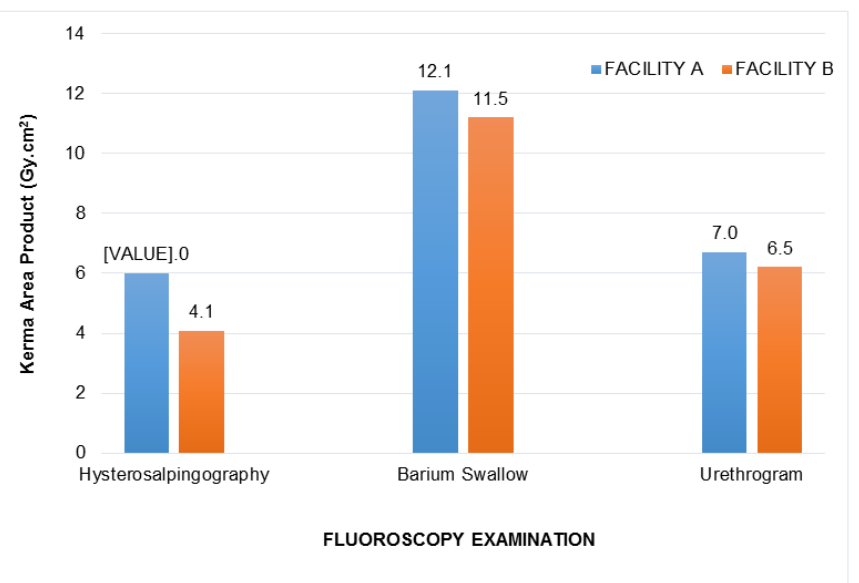

Figure 3 : Comparison of local diagnostic reference levels (LDRLs) for examination types at Facilities A and B.

Figure 3 shows the values of local diagnostic reference levels for this study compared to each other. From figure 3 above, Hysterosalpingography examinations recorded the lowest DRL values followed by urethrogram and barium swallow examinations respectively. At facility A, DRLs for HSG examinations were lower than barium and urethrogram examinations by a factor of 2.0 and 1.2 respectively, whiles at facility $\mathrm{B}$ the factors were 2.8 and 1.5 . The relatively high-calculated DRL values recorded for barium swallow can be attributed to the prolonged fluoroscopic screening time and complexity of the examination.

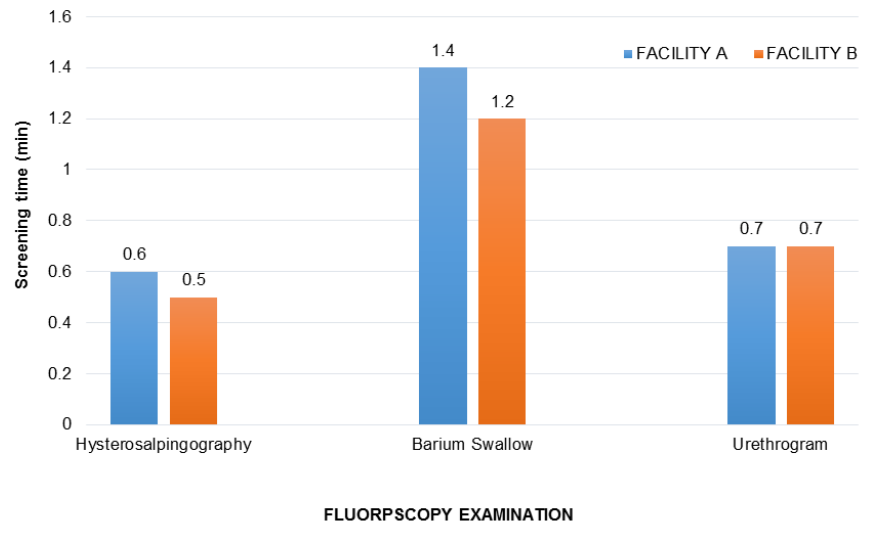

Figure 4. Comparison of diagnostic reference levels (DRLs) of fluoroscopy screening time per examination at Facilities A and B.

From figure 4, a comparison of DRLs for the fluoroscopy screening time per examination at both facilities was done. The recorded results of screening times for the different examination types indicated that HSG examinations had shorter screening time than $\mathrm{BaS}$ and urethrogram examinations. At facility $\mathrm{A}$, the screening time were shorter for HSG examinations by a factor of 2.3 and 1.2 for barium swallow and urethrogram examinations respectively. At facility B, it was lower by a factor of 2.4 and 1.4 for barium swallow and urethrogram examinations respectively. Higher DRL screening time values recorded for the Barium swallow studies can be attributed to the complexity of the examination since other studies have shown that the screening time of the fluoroscopy procedure is proportional to its complexity.

Table 2: Comparison of diagnostic reference levels (DRLs) of KAP values for Hysterosalpingography and barium swallow examinations for this study with Kenya and UK study 


\begin{tabular}{|c|c|c|c|c|}
\hline \multirow[t]{3}{*}{ EXAM } & \multicolumn{3}{|c|}{$\begin{array}{l}\text { DRLs } \\
{\left[\operatorname{KAP}\left(G y \cdot \mathrm{cm}^{2}\right)\right]}\end{array}$} & \multirow[b]{2}{*}{$\begin{array}{l}\text { Hart et al, } \\
2012\end{array}$} \\
\hline & $\begin{array}{l}\text { Facility } \\
\text { A }\end{array}$ & Facility B & $\begin{array}{l}\text { Wambani } \\
\text { et al, } 2014\end{array}$ & \\
\hline & & & Kenya Study & UK Study \\
\hline HSG & 6.0 & 4.1 & 3.0 & 2.0 \\
\hline $\begin{array}{l}\text { Barium } \\
\text { Swallow }\end{array}$ & 12.1 & 11.5 & 9.0 & 7.5 \\
\hline $\begin{array}{l}\text { Urethrog } \\
\text { ram }\end{array}$ & 7.0 & 6.50 & - & - \\
\hline
\end{tabular}

From Table 2, A comparison of DRLs of KAP values for hysterosalpingography, barium swallow and urethrogram examinations for this study with other studies was done. LDRLs values obtained at Facilities $A$ and $B$ for the three examinations were within the values obtained by international accepted DRLs. However, the DRL values at Kenyatta National Hospital [9] and DRLs of UK [10] have lower values as compared to the values of this study. Wambani et al., obtained values that were lower than facilities $A$ and B by a factor of 2 and 1.4 for HSG examination respectively. Hart et al., DRL value was also lower than this study by a factor of 3 and 2.1. For Urethrogram examination, there was no available data for comparison. The high DRL KAP values of 12.1 Gy.cm² and 11.5 Gy.cm² for barium swallow recorded for this research study indicates that the fluoroscopy examinations were performed with higher doses, resulting in high DRLs for the KAP values. The DRLs from the fluoroscopy examinations in this study compared to International Diagnostic Reference levels (IDRLs) indicates the need for proper training in, as well as the use of optimized imaging techniques and protocols. The similar trends in results for KAP values for both facilities in this study also suggest that anatomical-related imaging techniques and protocols to be standardised at the two facilities. The standardization and benchmarking of protocols and procedures must be continually improved for optimization purposes. Standardization of protocols should be established to outline the number of images acquired per examination to a complete procedure by a radiologist.

Table 3: Comparison of diagnostic reference levels (DRLs) for Fluoroscopy Screening Time for Hysterosalpingography, Barium swallow and Urethrogram examinations for this study with Kenyatta National Hospital and UK study

\begin{tabular}{lllll}
\hline EXAM & \multicolumn{3}{l}{$\begin{array}{l}\text { DRLs } \\
\text { ( Fluoroscopy Screening Time (minutes) })\end{array}$} \\
\hline & Facility A & Facility B & $\begin{array}{l}\text { Wambani } \\
\text { et al, 2014 }\end{array}$ & $\begin{array}{l}\text { Hart et al, } \\
2012\end{array}$ \\
& & & & \\
\hline HSG & 0.60 & 0.50 & 2.10 & 0.70 \\
$\begin{array}{l}\text { Barium } \\
\text { Swallow }\end{array}$ & 1.40 & 1.20 & 2.6 & 2.1 \\
& & & & \\
Urethrogram & 0.70 & 0.70 & - & - \\
\hline
\end{tabular}

The DRLs values for fluoroscopy screening time for Hysterosalpingography and Barium swallow examinations for this research work were compared with Kenyatta National Hospital [9] and UK study [10]. It was observed that, the DRL values for the HSG examination screening time for this study, was lower compared with that of Kenyatta National Hospital [9] but close to that of UK study [10]. The Kenyatta National Hospital had a higher value for HSG examination than that of facilities A and B by a factor of 3.5 and 4.2 respectively. For Barium swallow examinations, facilities $A$ and $B$ recorded lower values compared to Kenyatta hospital by a factor 2.0 and 1.5 [9] and was lower to UK studies values [10] by a factor of 2.3 and 1.8 respectively. It can be noted that this research work and UK study had a shorter screening time during the examination procedure, therefore resulting in the lower DRL values obtained for the examinations. Moreover, due to the 
unavailability of screening time for Urethrogram examination from other studies used, comparison was not done.

\section{IV.CONCLUSION}

This research observed a slight variation of values observed across facilities and can be attributed mainly to difference in protocols and techniques used in the two facilities. Similarly reported LDRLs values obtained at facilities $A$ and $B$ for the three examinations were within the values obtained by international accepted DRLs. However, it was noted that the DRL KAP values at Kenyatta National Hospital and UK study have lower values as compared to the values of this research work. Whiles the DRLs for the screening time values obtained for both Kenyatta National Hospital and UK study had higher values.

Generally, factors that affect patient doses for fluoroscopy examinations could be attributed to the radiological technique, the screening time and the number of images taken during the procedure. Hence, radiographers and radiologists should use optimized technique factors to improve and strengthen the protection of patients by minimizing the screening time used during a fluoroscopy procedure. Again, this work suggests standardization of protocols across facilities as a means to increase optimization of doses in order to limit observed variations in KAP and screening time values for the same type of examination.

\section{ACKNOWLEDGEMENT}

The authors wish to express my sincere thanks to the radiographers of the various facilities where data was collected for their immerse contribution and support.

\section{REFERENCES}

[1] Gingold, E. (2014). Modern Fluoroscopy Imaging Systems. Image Wisely.

[2] Aroua, A., Rickli, H., Stauffer, J. C., Schnyder, P., Trueb, P. R., Valley, J. F., \& Verdun, F. R. (2007). How to set up and apply reference levels in fluoroscopy at a national level. European radiology, 17(6), 1621-1633.

[3] Foley, S. J., McEntee, M. F., \& Rainford, L. A. (2012). Establishment of CT diagnostic reference levels in Ireland. The British journal of radiology, 85(1018), 1390-1397.

[4] Erskine, B. J., Brady, Z., \& Marshall, E. M. (2014). Local diagnostic reference levels for angiographic and fluoroscopic procedures: Australian practice. Australasian physical \& engineering sciences in medicine, 37(1), 75-82.

[5] International Commission on Radiological Protection, (2007). The 2007 Recommendations of the International Commission on Radiological Protection. ICRP Publication 103, Ann. ICRP 37 (2-4).

[6] Addo, P. (2016). Computed Tomography (CT) radiation dose in children: A survey to propose regional diagnostic reference levels in Greater Accra-Ghana.

[7] Gyasi, E. (2013). Assessment of radiation dose due to fluoroscopic procedures in patients at some selected facilities in the Greater Accra Region, Ghana.

[8] Vañó, E., Miller, D. L., Martin, C. J., Rehani, M. M., Kang, K., Rosenstein, M., \& Rogers, A. (2017). ICRP Publication 135: Diagnostic Reference Levels in Medical Imaging. Annals of the ICRP, 46(1), 1-144.

[9] Wambani, J. S., Korir, G. K., Tries, M. A., Korir, I. K., \& Sakwa, J. M. (2014). Patient radiation exposure during general fluoroscopy examinations. Journal of applied clinical medical physics, 15(2), 262-270. 
[10] Hart, D., Hillier, M. C., \& Shrimpton, P. C. (2012). Report HPA-CRCE-034: doses to patients from radiographic and fluoroscopic $\mathrm{x}^{-}$ ray imaging procedures in the UK-2010 review. Health Protection Agency, Chilton, 3.

\section{Cite this article as :}

Otoo J, Boadu M, Sosu E, Inkoom S, "Patient Radiation Dose Assessment during Fluoroscopic Procedures: A Survey to Propose Local Diagnostic Reference Levels for Selected Facilities", International Journal of Scientific Research in Science and Technology (IJSRST), Online ISSN : 2395-602X, Print ISSN : 2395-6011, Volume 7 Issue 4, pp. 05-11, JulyAugust 2020. Available at doi $\quad$ : https://doi.org/10.32628/IJSRST207378 Journal URL : http://ijsrst.com/IJSRST207378 